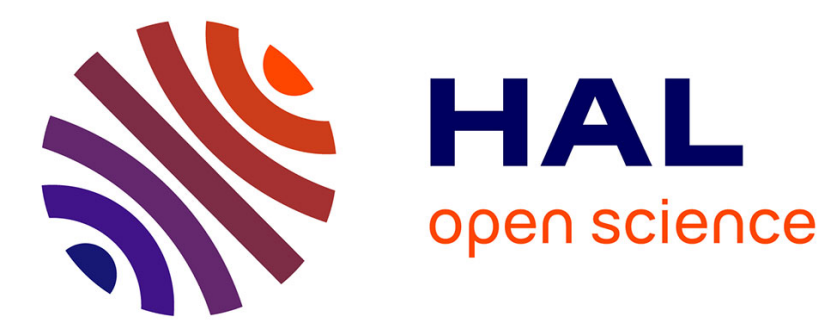

\title{
L'impossible rupture de Gerhard Schröder
}

Anne-Marie Le Gloannec

\section{To cite this version:}

Anne-Marie Le Gloannec. L'impossible rupture de Gerhard Schröder. Critique Internationale, 1999, 2, pp.29-33. 10.3406/criti.1999.1536 . hal-01010718

\section{HAL Id: hal-01010718 https://hal-sciencespo.archives-ouvertes.fr/hal-01010718}

Submitted on 20 Jun 2014

HAL is a multi-disciplinary open access archive for the deposit and dissemination of scientific research documents, whether they are published or not. The documents may come from teaching and research institutions in France or abroad, or from public or private research centers.
L'archive ouverte pluridisciplinaire HAL, est destinée au dépôt et à la diffusion de documents scientifiques de niveau recherche, publiés ou non, émanant des établissements d'enseignement et de recherche français ou étrangers, des laboratoires publics ou privés.

\section{(a)(1) $\$$}

Distributed under a Creative Commons Attribution - NonCommercial - NoDerivatives| 4.0 


\section{L'impossible rupture de Gerhard Schröder}

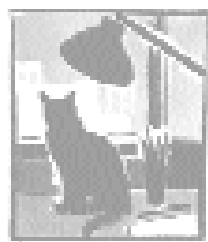

Contre-jour

par Anne-Marie Le Gloannec

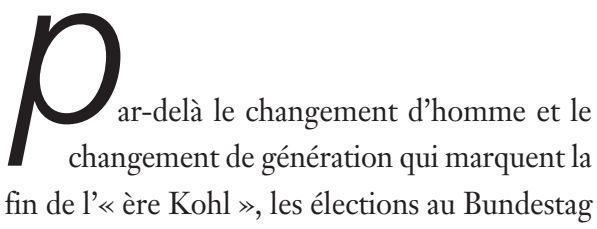

du 27 septembre 1998 sont remarquables à plus d'un titre. Tout d'abord, parce qu'elles consacrent le premier changement de gouvernement depuis la réunification. Ensuite, parce qu'elles permettent à une coalition inédite, rouge et verte, de parvenir au pouvoir. Enfin, fait moins souvent relevé, parce qu'elles entérinent la mort du tripartisme qui avait prédominé pendant plus de trente ans. Cette triple modification du paysage politique reflète un changement profond de la société allemande, une diversification résultant de la réunification de l'Allemagne et de l'installation durable de populations étrangères. Moins homogène que naguère, la République fédérale risque sinon d'être plus difficile à gouverner, du moins de ne plus être dominée par de grands partis populaires qui ne parviennent plus guère à satisfaire des électorats émiettés. La coalition rouge-verte, tiraillée entre gauche et centre, entre tradition ouvrière, revendications écologistes et libéralisme économique, risque d'en faire la difficile expérience.

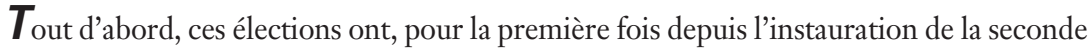
République, consacré la défaite du gouvernement sortant. L'ensemble des analyses montrent qu'avant toute chose, ce vote fut un vote-sanction. La majorité des électeurs souhaitaient un nouveau visage, un nouveau chancelier, comme l'ont montré tous les sondages. Le SPD de Gerhard Schröder recueillit 40,9 \% des voix, 5 points de plus que la CDU du chancelier Kohl. Au-delà du changement d'hommes cependant, on a voulu célébrer la « normalisation » du jeu démocratique, la « confiance en soi des citoyens » allemands qui prennent en main leur destinée, pour citer la formule de Jürgen Habermas (dans Die Zeit, 8 octobre 1998), l'affaiblissement de l'emprise partisane, puisque seuls les partis avaient jusqu'alors défait les coalitions et les chanceliers. Au début des années soixante, Adenauer avait été poussé à la porte par sa propre coalition. En 1974, Willy Brandt avait dû céder le pouvoir sur les instances de son parti et, quelques années plus tard, en 1982, Helmut Schmidt fut lâché par ses partenaires libéraux et par la base de son parti. Il fallut au demeurant recourir à une manipulation de la Constitution pour permettre son départ et la nomination d'Helmut Kohl à la tête d'un nouveau gouvernement. On eut 
beau jeu de critiquer, par la suite, le Parteienstaat, l'État partisan, et la toute-puissance des partis, accusés d'étouffer la démocratie. Ce n'est qu'avec les élections de 1998 que les Allemands écartent un chancelier dont ils ne veulent plus et élisent un homme plutôt qu'une coalition gouvernementale « prête-à-porter ».

Car, en effet, les Allemands ne voulaient guère d'une coalition rouge-verte. Celle-ci résulte de l'addition des voix et non d'un calcul délibéré des électeurs alors que, traditionnellement, ceux-ci votaient d'abord pour une coalition avant de voter pour un parti. À l'appui de cette thèse, certains instituts spécialisés dans l'analyse des comportements électoraux arguent qu'avant les élections, une coalition rouge-verte n'avait les faveurs que de $36 \%$ d'interrogés tandis que $49 \%$ y étaient opposés, dont $22 \%$ d'électeurs du SPD. Toujours est-il qu'au lendemain des élections, l'atmosphère était sobre : point de manifestations semblables à celles qui avaient accompagné l'élection de Willy Brandt en 1969 ou en 1972 ou de célébrations comme celles qui eurent lieu en France en 1981. On peut par ailleurs remarquer que le SPD a réalisé un score inférieur à celui des années soixantedix et quatre-vingt : 40,9\% contre un peu plus de $42 \%$ aux élections de 1969, de 1976 et de 1980 et près de $45 \%$ aux élections triomphales de 1972, véritable plébiscite en faveur de Willy Brandt qui allait pourtant quitter le pouvoir deux ans plus tard. Le SPD auraitil gagné par défaut, et faut-il plutôt parler de défaite de la CDU ?

Au risque de simplifier, on notera que la social-démocratie l'a emporté dans les anciens Länder, notamment auprès des travailleurs syndiqués (dont elle a remporté 61 \% des voix contre $48 \%$ des voix des non-syndiqués) et des employés (naguère partisans de la démocratie chrétienne), sans prévaloir véritablement dans les nouveaux Länder (y gagnant certes plus de 10 points par rapport à 1994 contre 6,6 points à l'Ouest, tout en ne remportant que $35 \%$ des voix à l'Est contre plus de $42 \%$ à l'Ouest) ni progresser en voix parmi ceux qui votaient pour la première ou la seconde fois. $\mathrm{La} C D U$, au contraire, a perdu des voix dans toutes les catégories socio-professionnelles, elle a vu son écho auprès des femmes - traditionnellement chrétiennes-démocrates mais spécifiquement touchées par la réduction des retraites - baisser de plus de $60 \%$, et elle a véritablement perdu les élections dans les nouveaux Länder, alors qu'elle les y avait remportées en 1994 (passant de près de 42 \% des voix en 1994 à 27,4 \% en 1998). Il n'en demeure pas moins que le SPD a su convaincre une bonne partie de la population de sa compétence économique, notamment en ce qui concerne la lutte contre le chômage et son désir de préserver la protection sociale, gagnant des voix dans toutes les catégories socio-professionnelles, à l'Est comme à l'Ouest.

Néanmoins, certaines couches de la population ont tourné le dos aux grands partis populaires, démocratie chrétienne et social-démocratie : ainsi les jeunes, qui ont plus volontiers donné leurs voix aux petits partis, verts à gauche et DVU ou NPD à droite, ou encore les électeurs des nouveaux Länder. Ceux-ci ont en effet déserté la CDU sans se tourner vers le SPD dans les mêmes proportions, votant en faveur de l'extrême droite plus que leurs contemporains de l'Ouest (5,3 \% à l'Est contre 2,9\% à l'Ouest), et surtout en faveur du PDS, héritier du SED communiste (auquel près de $22 \%$ des Allemands de l'Est ont 
donné leur voix, contre $1 \%$ de ceux de l'Ouest). Pour la première fois, celui-ci a pu entrer au Parlement en passant la barre des $5 \%$, alors que sa présence antérieure y était due à des mandats directs. Le PDS pérennise ainsi sa présence sur la scène politique allemande, sanctionnant la transformation du système des partis.

Tripartisan trente ans durant, de la fin des années cinquante aux années quatre-vingt, lorsque le FDP libéral faisait et défaisait les coalitions en s'alliant à l'un des deux grands partis populaires, le système a entamé une longue mutation dans les années quatre-vingt, avec l'entrée des Verts au Parlement. Dans les années quatre-vingt-dix, la transformation s'est confirmée avec, d'une part, l'« embourgeoisement » des Verts, qui en quelque quinze ans ont acquis une respectabilité telle qu'ils peuvent détrôner le FDP comme partenaire de coalition (sans que celui-ci disparaisse pour autant) et, d'autre part, avec la consolidation du PDS dans l'ex-RDA. Parallèlement, les grands partis populaires, CDU mais aussi SPD, n'ont cessé de perdre des fidèles, membres et électeurs, un peu comme s'ils ne pouvaient satisfaire une clientèle désormais diversifiée, suivant un modèle de consommation où les produits de masse ne font plus recette. Dans les créneaux ainsi libérés s'insèrent des partis proches des groupes d'intérêt, ou des lobbies, comme le PDS, dont ses électeurs est-allemands disent qu'il est le seul à représenter les intérêts de l'ancienne RDA et à traduire sa spécificité. Avec la multiplication des partis politiques, cinq désormais, un plus grand nombre de combinaisons gouvernementales s'avère possible : le système se fluidifie en quelque sorte, perdant de cette stabilité qui avait fait à la fois sa force - celle de gouvernements solides - et sa faiblesse - celle du Parteienstaat, dénoncé plus haut - quelques décennies durant.

Cette transformation du système partisan, sinon du système politique, reflète une diversification de la société allemande depuis les années quatre-vingt et, plus encore, quatrevingt-dix. D’une part, la République fédérale s'est agrandie de quelque dix-sept millions d'habitants dont, on ne l'a que trop dit, la socialisation s'est opérée dans des conditions radicalement différentes de celle des citoyens ouest-allemands. De fait, la greffe des partis politiques venus de l'Ouest n'a pas pris en ex-RDA, pour l'heure du moins. Les grands partis populaires, $\mathrm{CDU}$ et $\mathrm{SPD}$, y sont très mal implantés, perdant des membres déjà peu nombreux, et le FDP y est inexistant. Seul le PDS possède une solide infrastructure, matérielle et humaine, héritage de l'ancien parti communiste qu'il a su consolider, unique réseau subsistant dans cet ensemble anomique que sont les nouveaux Länder : à cet égard, il se substitue en quelque sorte aux réseaux de l'ancien régime, qui prenaient en charge, par le biais des entreprises notamment, les activités des salariés. Des partis d'extrême droite, il n'y a que le NPD pour exercer une fonction analogue d'encadrement alors que la DVU est une sorte de parti virtuel, qui a remporté les élections de Saxe-Anhalt au printemps 1998 sans être présent sur le terrain, par la seule grâce du courrier, traditionnel et électronique. De fait, les Allemands de l'Est croient plus volontiers que ceux de l'Ouest 
aux vertus de l'État et à son intervention dans l'économie et la société - l'inexistence du Parti libéral dans les nouveaux Länder tendrait d'ailleurs à le prouver.

D'autre part, la République fédérale a changé par sa population immigrée. Si la venue de travailleurs temporaires (Gastarbeiter) s'interrompt au milieu des années soixante-dix, la population immigrée durablement installée en Allemagne, en particulier turque, mais aussi grecque, italienne et autre, s'accroît dès lors grâce au regroupement familial. Dans les années quatre-vingt viennent s'y ajouter des demandeurs d'asile, avant la modification de la Loi fondamentale notamment, et des réfugiés venant surtout de l'ancienne Yougoslavie. Le vocabulaire reflète au demeurant ces transformations, puisque le terme de Gastarbeiter a fait place à celui de ausländische Mitbürger, concitoyens étrangers. Si la population étrangère n'a, bien entendu, pas voté, il n'en est pas moins vrai qu'on a en quelque sorte voté pour elle : dans la mesure où SPD et Verts promettaient de modifier la loi sur l'acquisition de la nationalité, ceux qui prenaient en compte cette proposition dans leur choix électoral votaient pour les quelque trois millions d'étrangers susceptibles d'acquérir la nationalité allemande.

Ce second changement a et aura des conséquences profondes sur la société allemande, non seulement sur le paysage urbain, mais aussi sur la manière dont cette société se voit elle-même. S'il est trop tôt pour en évaluer les répercussions sur le système partisan, voire politique - sur un éventuel vote à gauche des nouveaux citoyens, sur leur insertion dans la cité etc. -, on peut en revanche souligner que la réunification paraît remettre en cause certains principes du fédéralisme, déjà critiqué dans son fonctionnement depuis plusieurs années. En effet, les Länder de l'Est grèvent les finances publiques et les plus riches des États allemands, Bavière et Bade-Wurtemberg, appuyés par la Basse-Saxe, contestent devant la Cour constitutionnelle la péréquation financière sous sa forme actuelle. Par-delà cette question se pose celle du fonctionnement des institutions. La Constitution de la République fédérale stipule en effet que Bund et Länder ont à la fois des compétences exclusives et des compétences conjointes. Or celles-ci ont crû immodérément, notamment depuis les années soixante-dix, de sorte que le Bundesrat, chambre non élue où sont représentés les parlements régionaux, prend une part de plus en plus importante dans le processus législatif.

Dans les mois précédant les élections, des voix s'étaient élevées pour réclamer notamment une division plus verticale des compétences entre Bund et Länder, à l'instar de la division des pouvoirs entre État fédéral et États fédérés aux États-Unis, par le biais d'une distinction claire entre impôts perçus par le Bund et impôts dévolus aux Länder : Theo Waigel, l'ancien ministre des Finances, et Wolfgang Schäuble, chef de la fraction parlementaire, s'en étaient faits les avocats. La nouvelle coalition gouvernementale saura-telle opérer de telles réformes ? Ou choisira-t-elle la facilité du fait qu'elle dispose d'une majorité au Bundesrat comme au Bundestag, ce qui lui permettrait de légiférer sans réformer ? La frilosité des propositions mises en avant lors des négociations de coalition précédant la formation du gouvernement augurait mal des réformes futures. En matière 
d'impôts en particulier, la réforme tant attendue semblait devoir se réduire à quelques modifications marginales. Loin de bouleverser le système fiscal, la coalition rouge-verte ne proposait que des corrections : modification des tranches imposables (et encore, pour ce qui est de l'impôt sur le revenu, la tranche imposable maximale devait être abaissée d'un petit demi-point, ultime concession d'un Oskar Lafontaine en l'occurrence plus « gauchiste » que les Verts eux-mêmes !) et suppression d'exemptions, sans qu'aucun grand souffle réformateur ne vînt débrouiller un système extrêmement complexe, ni que les entreprises vissent leurs charges véritablement allégées. D'une réforme du financement des retraites, il n'a pas non plus été question lors de ces négociations, alors que le système sera bientôt ingérable du fait de la pyramide des âges. Les partisans de réformes plus radicales en étaient, pour l'heure du moins, pour leurs frais, les entreprises en particulier, qui attendaient du gouvernement un allègement des charges sociales et une flexibilité plus grande en matière juridique. Certes les grandes entreprises ont, depuis la récession de 1993-1995, opéré des restructurations qui leur permettent de s'internationaliser mais les PME-PMI, sous-traitées pour certaines par les multinationales allemandes et qui, en tout état de cause, irriguent l'économie du pays, souffrent d'une inertie gouvernementale que la nouvelle coalition ne paraît pas vouloir corriger.

N'est-il pas paradoxal, en somme, que l'Allemagne ait voté pour le changement sans changement, pour un changement d'hommes sans changement véritable, sans réforme des politiques publiques et de la protection sociale? Des sondages récents montraient qu'une majorité de la population redoute des réformes, à l'Est plus encore qu'à l'Ouest. De sorte qu'on aurait voté à gauche pour maintenir le statu quo. En réalité, cette supposition ne vaut qu'à demi : une partie des électeurs de Schröder ont voté pour celui qui se faisait l'avocat du «nouveau milieu » (die neue Mitte), le héraut de l'économie allemande, celui qui voulait faire de Jost Stollmann, un entrepreneur freelance de la politique sans carte du parti, son ministre de l'Économie. L'alchimie des négociations gouvernementales en a décidé autrement : non que les Verts aient pesé bien lourdement sur celles-ci, étant donné la faiblesse relative de leur performance, inférieure aux attentes de la campagne. C'est du SPD lui-même qu'est venu le blocage, en la personne d'un Oskar Lafontaine, maître du parti, défendant une position de pouvoir à partir d'une politique dite de gauche. Interventionniste, partisan d'une politique fondée sur la demande plus que sur l'offre - et encore, puisque les allègements d'impôts sont bien moins importants que ceux proposés par le gouvernement sortant -, peu désireux d'élaguer des subventions qui pourtant alourdissent le budget fédéral, Oskar Lafontaine s'est taillé un super-ministère des Finances, empiétant sur les compétences du ministère de l'Économie dont Jost Stollmann n'a, pour finir, plus voulu. Le futur chancelier n'a pas su peser sur ces orientations politiques même s'il a étouffé les velléités d'Oskar Lafontaine de placer à la tête de la fraction parlementaire un homme de son choix. Gerhard Schröder saura-t-il à l'avenir répondre aux attentes de l'économie, ou se laissera-t-il emporter par la pesanteur du statu quo et par le désir de ne pas heurter de front le puissant Lafontaine? 